\title{
On the Minimum Communication Effort for Secure Group Key Exchange
}

\author{
Frederik Armknecht ${ }^{1}$ and Jun Furukawa ${ }^{2}$ \\ 1 Universität Mannheim, Germany \\ armknecht@informatik.uni-mannheim.de \\ 2 NEC Corporation, Japan \\ j-furukawa@ay.jp.nec.com
}

\begin{abstract}
Group key exchange protocols (GKE) allow a set of parties to establish a common key over an insecure network. So far the research on GKE mainly focused on identifying and formalizing appropriate security definitions that has led to a variety of different security models. Besides reaching a high security level, another important aspect is to reduce the communication effort. In many practical scenarios it is preferable (or possibly even indispensable) to reduce the number of messages to a minimum, e.g., to save time and/or energy.

We prove that any $n$-party GKE that provides forward security (FS) and mutual authentication (MA) against insider attackers needs at least two communication rounds and in that case at least $\frac{1}{2} n^{2}+\frac{1}{2} n-3$ messages. Observe that FS and MA are today accepted as basic security recommendations. Hence these bounds hold automatically as well for more elaborate security definitions.

Then, we describe a 2-round-GKE that requires $n+1$ messages more than the derived lower bound. We prove that the protocol achieves UC-security (in the model by Katz and Shin (CCS'05)) in the common reference string (CRS) model. To the best of our knowledge, this represents the most communication efficient (in terms of number of rounds and messages) UC-secure GKE so far.
\end{abstract}

Keywords: Group key exchange, communication effort, UC security.

\section{Introduction}

Many cryptographic mechanisms, especially for confidentiality and authenticity, require a common secret to be shared between the communication partners. Therefore, key exchange protocols (KE) belong to the most practically relevant cryptographic primitives. However, while several secure and efficient KEs are known and used nowadays for the case of $n=2$ parties, the situation becomes more complicated in the case of group key exchange protocols (GKE), where a key needs to be established between $n>2$ parties.

Obviously, an optimal GKE should achieve the highest security goal with the lowest possible communication effort. Here, the communication effort is usually represented in the number of communication rounds and the number of messages. However, only little is known about the minimum communication effort of GKEs. This question is not only of theoretical interest. In practice the exchange of messages between the parties can be more time consuming than the computations themselves. Thus, reducing the

A. Biryukov, G. Gong, and D.R. Stinson (Eds.): SAC 2010, LNCS 6544, pp. 320-337, 2011.

(C) Springer-Verlag Berlin Heidelberg 2011 
number of messages can help to reduce the time effort. Furthermore, the energy effort for sending messages (wireless) is usually by magnitudes higher than for performing computations. Therefore, GKEs with reduced communication effor 11 are beneficial for application scenarios where low-weight devices with restricted resources are involved.

Actually, the minimum communication effort depends on several factors. For example, although it is common to identify each protocol run by an individual session identity (SID), differences exist on how to set up the SID. In principle, one can distinguish between two fundamental approaches: external-SID GKEs, where a globally unique SID is provided by an external environment, and internal-SID GKEs, where a SID is determined by the group members themselves during the protocol execution. As we will see later this already impacts the minimum communication effort.

Other factors are the considered security model and security goals. Up to now, a variety of different security models for GKEs exist. Based on the two-party case [3|4]2], Bresson et al. [978] gave the first formal security model for GKEs. They formalized the notions of authenticated key exchange (AKE) and mutual authentication (MA). AKE means informally that a group key is computationally indistinguishable from a random string while MA guarantees that each party is assured of the participation of every other party in the protocol. Initially, these definitions considered outsider attackers only, that is, adversaries who do not take part in the protocol. Later on, Katz and Shin [20] introduced the notion of insider attacks: an attacker can corrupt several group members and can actively participate into the protocol. They presented a security model for GKEs within the Universal Composability (UC) framework [14] and showed that their model covers insider attackers. They also presented the construction of UC-secure GKE. A UC-secure protocol maintains its security properties even when composed concurrently with an unbounded number of instances of other UC-secure protocols. In [11], Bresson et al. revised the model from [9] so that (among other things) insider attackers are captured as well. Another important security notion is contributiveness that has been brought up by Bohli et al. [5] and strengthened by Bresson and Manulis [10]. A protocol satisfying this property ensures that a proper subset of insiders cannot predetermine the session key. Gorantla et al. [17] showed how to define UC-secure GKEs that provide contributiveness. Evidently, the search for a comprehensive security model for GKEs is still within the focus of current research, bringing up new security notions like key compromise impersonation (e.g., Gorantla et al. [16]) or the consideration of special approaches like password-authenticated GKEs (e.g., Abdalla et al. [1]).

Intuitively, one can expect that most additional security requirements on GKEs will not "come for free". Making a GKE stronger will probably increase the computational and/or communication effort. Previous works mostly investigated GKEs from a security perspective, asking the question: When is a GKE secure? Here, we approach GKEs more from complexity theory and ask: How much does it cost (in terms of messages and rounds) to make a GKE secure?

Actually, we are only aware of the work by Furukawa et al. [15] that addressed directly the question of the minimum communication effort of GKEs. More precisely

\footnotetext{
${ }^{1}$ Of course, the communication effort depends on the length of the messages as well. We do not investigate this question in this work.
} 
they proved that any 2-round UC-secure 2 GKE with internal-SID generation between $n$ parties requires at least $2 n^{2}-2 n$ messages. Observe that this bound relies on two rather strong assumptions: (i) UC-security (which requires straight-line simulatability) and (ii) internal-SID generation. Therefore, it is plausible to assume that both aspects impact the minimum communication effort but the question is to what extent.

Contribution. In this work we pick up the question investigated by Furukawa et al. [15]. First, we derive lower bounds on the communication effort of GKEs based on a selection of minimum security requirements only: forward secrecy (FS) (the corruption of a party has no impact on the security of past protocol runs) and mutual authentication (MA) (an honest participant outputs a key only if all other participants in this group agreed to participate in the GKE and all honest parties generate the same key) in the presence of insider attackers (attackers that actively participate in the protocol). To have a clear separation between the effort for SID generation and key establishment, we consider external-SID GKEs only. We show that under these conditions, GKEs require at least two communication rounds and in this case at least $\frac{1}{2} n^{2}+\frac{1}{2} n-3$ messages in total. Interestingly this bound is significantly smaller than the lower bound derived in [15]. This sheds some lights on the "costs" for fulfilling additional requirements (as UCsecurity and SID-generation in this case). To the best of our knowledge the notions of forward secrecy and mutual authentication against insider attackers are covered by any recent security models for GKEs. Thus, they can be seen as basic requirements and the lower bounds should be valid for any current or upcoming security mode 3 . We note that the number of messages might be smaller for GKEs that comprise more than two rounds. However, from our point of view, minimizing the number of rounds will have a more significant impact in reducing the time effort for GKEs than reducing the number of messages. The reason is that in practice, we expect that more time is spend for waiting for all messages within one round, i.e., waiting that a round is completed, than for generating the messages themselves. Therefore, minimizing the number of rounds was our primary focus.

Our second contribution is a 2 -round GKE that requires $\frac{1}{2} n^{2}+\frac{3}{2} n-2$ messages, being $n+1$ messages more than the derived lower bound. We prove that the protocol is UC-secure (based on the UC-model given by Katz and Shin [20]) in the common reference string model under the decisional Diffie-Hellman assumption. As UC-security implies FS and MA, the protocol illustrates that GKEs with almost optimal communication effort are possible. Of independent interest might be our observation that although we aim for "symmetric" security properties like mutual authentication, our protocol is highly asymmetric regarding the roles of the different participants. A further interesting observation is that both in the work by Furukawa et al. [15] and in our work, it is shown that one can construct GKEs that are UC-secure and have an almost optimal number of messages. This seems to indicate that considering UC-security has a rather minor impact on the minimum number of messages.

To the best of our knowledge, the most efficient (in number of messages) GKEs so far have been given by Furukawa et al. [15] and Gorantla et al. [17]. Both require 2 rounds

\footnotetext{
${ }^{2}$ If not mentioned otherwise, UC-secure refers to the Katz-Shin-model [20].

${ }^{3}$ Of course, the incorporation of additional assumptions might increase these bounds. We leave this question for future research.
} 
and $2 n^{2}-2 n$ messages, but possess stronger properties: internal SID-generation and contributiveness, respectively. This makes them rather incomparable but indicates again the higher communication effort for stronger requirements.

Organization. The paper is organized as follows: Sec. 2 informally repeats the common communication model and the security notions of internal attackers, forward secrecy (FS), and mutual authentication (MA). Sec. 3 presents some lower bounds on the communication effort of external-SID GKEs that provide forward secrecy and mutual authentication in the presence of insider attackers. Sec. 4 proposes a concrete GKE protocol that almost meets this lower bound and Sec.5 proves that it achieves UC-security. Sec. 6. concludes the paper.

\section{Preliminaries}

In this section we repeat some common aspects of GKE models. We assume for simplicity a fixed group of parties $\mathcal{P}$ of potential participants. A GKE is executed between the members of subset pid $=\left\{\Pi_{1}, \ldots, \Pi_{n}\right\} \subseteq \mathcal{P}$ where $\Pi_{i}$ is the party $I D$ of the $i$-th participant in the GKE. Each protocol execution is labeled by its own session ID (SID) sid that has to be globally unique. If a message (sid, pid, new-session) is send to an honest party $\Pi \in$ pid, a new instance ( $\Pi$, sid,pid) of $\Pi$ is invoked. An instance can be seen as a copy of $\Pi$ that has its own instance state (which, for example, stores all ephemeral values). All instances ( $\Pi$, sid,pid), $\Pi \in$ pid, start a new execution of a GKE $\pi$ where each instance uses its own instance state. During this session, every instance $\left(\Pi\right.$, sid, pid) communicates only with other instances $\left(\Pi^{\prime}\right.$, sid $^{\prime}$, pid $\left.^{\prime}\right)$ if $(\operatorname{sid}, p i d)=\left(s i d^{\prime}, p^{\prime} d^{\prime}\right)$, i.e., both instances participate into the same session sid with the same participants pid, and if $\Pi^{\prime} \in$ pid. If an instance $(\Pi$, sid, pid) finishes its participation into $\pi$, it outputs $(\Pi$, sid, pid, $\kappa)$ with $\kappa$ being the session key. Afterward, the instance $(\Pi$, sid, pid $)$ and its corresponding instance state is deleted.

As pointed out in Sec. 1, the definition of an appropriate security model for GKEs is still in the focus of current research. However, several requirements exist that are commonly agreed to be part of any meaningful security model. In the following, we single out some of these and explain them shortly. We refer to Appendix Afor a formal treatment of the considered security notions. We emphasize that the following list of conditions does not represent a full security model than rather a set of necessary basic requirements. In Sec. 3 we will show that these already imply some lower bounds on the communication effort. In consequence, the derived bounds automatically hold as well for any more elaborate security model that comprises the mentioned aspects.

An adversary can invoke a GKE between any subset pid $\subseteq \mathcal{P}$. In addition, she can control the communication within the network, and corrupt any party. Corruption means that she literally takes over control of the corrupted party and learns all its secrets. If corrupted parties participate into the protocol one speaks of insider attackers. Obviously, the secrecy of the key cannot be longer achieved in such cases but other security properties can still be true. One security requirement that is usually put on a GKE in the context of corruption is forward secrecy: if an attacker corrupts a party $\Pi$ at some point in time, this does not impact the security of past protocol runs which $\Pi$ participated to. Another established security requirement is mutual authentication: an honest party 


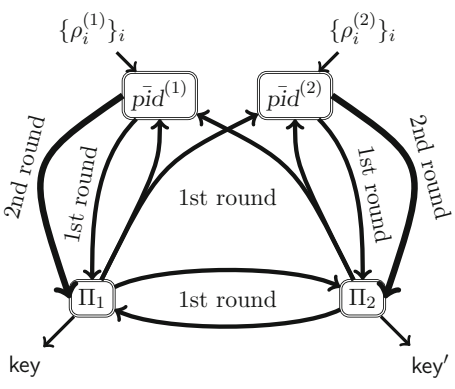

(a) Execution 1

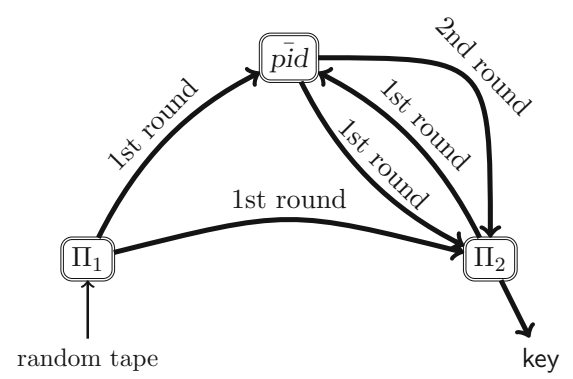

(b) Execution 2

Fig. 1. The protocol executions discussed in the proof of Theorem 1

only outputs a key if all parties in the same group confirmed their participation and keys generated by honest parties are equal.

\section{Lower Bounds on the Communication Effort}

In this section, we derive lower bounds on the number of rounds and the number of exchanged messages in GKEs that provide forward secrecy and mutual authentication in the presence of insider attackers. We prove our lower bounds by contradiction. We show that if the communication effort is below the specified bounds, then there exists either an insider adversary who violates mutual authentication or an outsider adversary who breaks forward secrecy.

Theorem 1. Consider a 2-round GKE between a group pid of parties that provides mutual authentication and forward secrecy in presence of insider attackers. For two different parties $\Pi, \Pi^{\prime} \in$ pid and $r \in\{1,2\}$ denote by $m_{\Pi \rightarrow \Pi^{\prime}}^{r}$ the message that $\Pi$ sends toward $4 \Pi^{\prime}$ in the $r$-th round. Furthermore, we define by $M_{\Pi, \Pi^{\prime}}^{r}:=\left\{m_{\Pi \rightarrow \Pi^{\prime}}^{r}, m_{\Pi^{\prime} \rightarrow \Pi}^{r}\right\}$ the set of messages exchanged between $\Pi$ and $\Pi^{\prime}$ in the $r$-th round. It holds:

1. If $M_{\Pi_{1}, \Pi_{2}}^{2}=\emptyset$ for two different honest parties $\Pi_{1}, \Pi_{2} \in$ pid, that is, $\Pi_{1}$ and $\Pi_{2}$ do not exchange any message in the second round, then the session key depends only on the random tapes of these two parties and some other public data, e.g., the session identity, the public keys of parties, etc.

2. There exists at most one pair $\left(\Pi_{1}, \Pi_{2}\right)$ of honest parties such that $M_{\Pi_{1}, \Pi_{2}}^{2}=\emptyset$.

Proof. The case of $n=2$ parties is straightforward. There is only one possible pair of parties so that the claims are obviously true. Hence, we restrict to the case $n \geq 3$ in the following.

\footnotetext{
${ }^{4}$ Recall that it is not guaranteed that the message eventually reaches $\Pi^{\prime}$ as the attacker is assumed to have complete control over the network.
} 
We describe for each claim a possible protocol execution (termed execution 1 and 2 and displayed in Fig. (1) where we show that if the claim is not fulfilled, the assumptions are violated. We start with the first claim and explain execution 1 . Let $\Pi_{1}, \Pi_{2} \in$ pid be two different honest parties in a 2-round GKE with $M_{\Pi_{1}, \Pi_{2}}^{2}=\emptyset$. We consider an adversary $\mathcal{A}$ who corrupts all parties in $\overline{\text { pid }}:=$ pid $\backslash\left\{\Pi_{1}, \Pi_{2}\right\}$. For each $\Pi_{i} \in \overline{\text { pid }}$, the adversary simulates two instances of $\Pi_{i}$, called $\Pi_{i}^{(1)}$ and $\Pi_{i}^{(2)}$. These use two independent random tapes, denoted by $\rho_{i}^{(1)}$ and $\rho_{i}^{(2)}$, respectively. Let $\overline{p i d}^{(1)}$ denote the set of all instances $\Pi_{i}^{(1)}$ for $i \geq 3$, and define analogously $\overline{\text { pid }}^{(2)}$.

The core idea of the proof is to show that the adversary $\mathcal{A}$ who controls the communication within the network can execute the protocol such that $\Pi_{1}$ receives messages only from $\overline{\text { pid }}^{(1)} \cup\left\{\Pi_{2}\right\}$ while $\Pi_{2}$ receives messages only from $\overline{\text { pid }}^{(2)} \cup\left\{\Pi_{1}\right\}$. We explain now in more detail how each message is handled.

Each message from either $\Pi_{1}$ or $\Pi_{2}$ to any other party $\Pi_{i} \in \overline{\text { pid }}$ is forwarded to both instances $\Pi_{i}^{(1)}$ and $\Pi_{i}^{(2)}$. However, the other way around, only messages from $\overline{\text { pid }}^{(1)}$ to $\Pi_{1}$ and from $\overline{\text { pid }}^{(2)}$ to $\Pi_{2}$ are forwarded while both messages from $\overline{\text { pid }}^{(1)}$ to $\Pi_{2}$ and messages from $\overline{\text { pid }}^{(2)}$ to $\Pi_{1}$ are dropped. All messages between $\Pi_{1}$ and $\Pi_{2}$ are forwarded. All messages from $\overline{\text { pid }}^{(1)}$ to $\overline{\text { pid }}$ are only sent to the corresponding instance

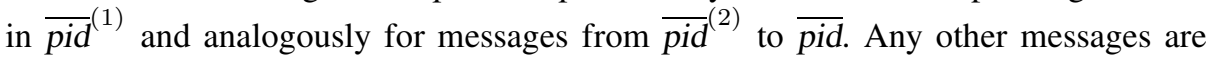
deleted. Execution 1 is displayed in Figure 1(a)

Observe that $\Pi_{1}$ communicates only with the instances in $\overline{p i d}^{(1)} \cup\left\{\Pi_{2}\right\}$, and similarly $\Pi_{2}$ only with $\overline{\text { pid }}^{(2)} \cup\left\{\Pi_{1}\right\}$. Recall that all parties in $\overline{\text { pid }}$ are under the control of the adversary. Hence, he can force the instances $\Pi_{i}^{(1)}, \Pi_{i}^{(2)}$ to use the same session ID sid as $\Pi_{1}$ and $\Pi_{2}$. Furthermore note that $\Pi_{1}$ and $\Pi_{2}$ communicate with different party instances $\overline{\text { pid }}^{(1)}$ and $\overline{\text { pid }}^{(2)}$ which use different random tapes $\rho_{i}^{(1)}$ and $\rho_{i}^{(2)}$ respectively. As $\Pi_{1}$ and $\Pi_{2}$ exchange only messages in the first round, the communication between $\Pi_{1}$ and $\Pi_{2}$ does not depend on any message from $\overline{p i d}$. Hence, they are not able to notice that each of them communicated with different instances from $\overline{p i d}$.

Now, by assumption the protocol provides mutual authentication which implies that all (honest) parties generate the same session key. Hence, the key has to be independent of the random tapes of $\overline{p i d}$ which shows the first claim.

Regarding the second claim, assume two different pairs of parties $\left(\Pi_{1}, \Pi_{2}\right)$ and $\left(\Pi_{1}^{\prime}, \Pi_{2}^{\prime}\right)$ who have no direct communication in the second round. Then it follows from claim 1 that either $\left\{\Pi_{1}, \Pi_{2}\right\} \cap\left\{\Pi_{1}^{\prime}, \Pi_{2}^{\prime}\right\} \neq \emptyset$ or that the exchanged key is independent of the random tapes of all parties. The latter case contradicts the assumption that the protocol provides forward secrecy.

Hence, we can assume w.l.o.g. that $\left\{\Pi_{1}\right\}=\left\{\Pi_{1}, \Pi_{2}\right\} \cap\left\{\Pi_{1}^{\prime}, \Pi_{2}^{\prime}\right\}$. This means in particular (with the same arguments as above) that the key depends only on the random tape of $\Pi_{1}$. Consider now execution 2 in which all messages are honestly forwarded by the adversary except of the following ones, which are discarded:

1. $\left\{m_{\Pi_{i} \rightarrow \Pi_{1}}^{1}\right\}_{i \geq 2}$ (all messages from pid $\backslash\left\{\Pi_{1}\right\}$ to $\Pi_{1}$ in the first round)

2. $M_{\Pi_{i}, \Pi_{1}}^{2}, i \geq 3$ (all messages between pid $\backslash\left\{\Pi_{1}, \Pi_{2}\right\}$ and $\Pi_{1}$ in the second round)

3. $\left\{m_{\Pi_{2} \rightarrow \Pi_{i}}^{2}\right\}_{i \geq 3}$ (all messages from $\Pi_{2}$ to pid $\backslash\left\{\Pi_{1}, \Pi_{2}\right\}$ in the second round) 
This execution is illustrated in Figure 1(b). In this execution, $\Pi_{2}$ cannot notice that some of messages are dropped since it receives only messages that are independent of the dropped messages. More precisely, $\Pi_{2}$ cannot notice that the messages $\left\{m_{\Pi_{i} \rightarrow \Pi_{1}}^{1}\right\}_{i \geq 2}$ are deleted as it has no communication with $\Pi_{1}$ in the second round by assumption. Furthermore, as none of the messages specified in 2. and 3. are addressed for $\Pi_{2}$ and as the protocol has in total two rounds, $\Pi_{2}$ cannot observe that these messages have been dropped. Concluding, from $\Pi_{2}$ 's point of view, the execution was correct and it outputs the correct session key at the end of the execution by assumption.

We also observe that $\Pi_{1}$ receives no messages from other parties and that all messages $\Pi_{1}$ sends in the second round are dropped. That means, an adversary can copy all the messages $\Pi_{1}$ sends in the first round of one session and later, even after the session is over (after the key is deleted), the adversary can corrupt pid $\backslash \Pi_{1}$ and recover the key exchanged in the session as $\Pi_{2}$ is able to compute the correct session key (as argued above). This contradicts forward secrecy, showing the claim.

Theorem 2. In the first round of a 2-round GKE $\pi$ with $n$ parties that achieves forward secrecy and mutual authentication in respect to insider attackers, at least $n-2$ messages are sent.

Proof. In the case of $n=2$ parties, this is obviously true as less than $n-2=0$ messages are impossible. We consider the case of $n \geq 3$ parties in the following. We first borrow some vocabulary from graph theory (see [6] for a reference). A path is a sequence of vertices such that there is an edge between two consecutive vertices. Two vertices $u$ and $v$ in a graph are connected if the graph contains a path from $u$ to $v$. Otherwise, they are disconnected. A connected component of a graph is a maximal subgraph in which any two vertices are connected to each other. Obviously each vertex belongs to exactly one connected component.

We consider parties of a GKE as vertices of a graph $\mathcal{G}$ and define an (undirected) edge between two parties $\Pi_{i}$ and $\Pi_{j}$ if $\Pi_{i}$ sends a message directly to $\Pi_{j}$ in the first round or vice versa. Then, there exists a unique (up to re-ordering) partition of the set

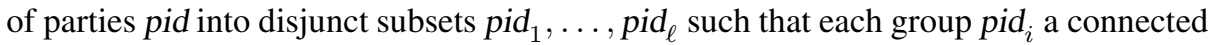
component of $\mathcal{G}$.

In case of $\ell=3$, we construct an imaginary GKE $\bar{\pi}$ out of $\pi$ as follows. We consider each group $\operatorname{pid}_{1}, \mathrm{pid}_{2}$, and $\mathrm{pid}_{3}$ as a party in $\bar{\pi}$. For each $i$, the random tape of $\mathrm{pid}_{i}$ is the concatenation of the random tapes of all parties $\Pi \in$ pid $_{i}$. If $\Pi \in$ pid $_{i}$ sends a

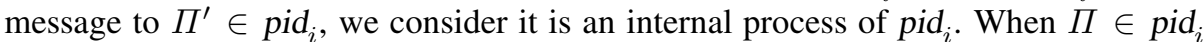
sends a message to $\Pi^{\prime} \in$ pid $_{j} \neq$ pid $_{i}$, we define that pid ${ }_{i}$ sends the message to $\mathrm{pid}_{j}$. When some parties in pid $_{i}$ output keys, we choose the first key that was given out and define it as being the key generated by $\operatorname{pid}_{i}$. When $\Pi \in$ pid $_{i}$ is corrupted, the whole set $\operatorname{pid}_{i}$ is corrupted as a party.

Since the parties pid $_{1}$, pid 2 , and pid $_{3}$ are by definition different connected components, they are in particular pairwise disconnected. That is, no messages are exchanged in the first round of $\bar{\pi}$. Hence, we can ignore the first round of the original GKE and consider $\bar{\pi}$ as a 2 -round GKE in which participants send no messages in the second round. In particular, pid $_{1}$ and pid $_{2}$ do not exchange any messages in the second round of $\bar{\pi}$. This implies that, as discussed in the proof of Theorem 1 , the session key does not depend on the random tape of pid $_{3}$. By the same argument, the session key does not 
depend on the random tapes of $\mathrm{pid}_{1}$ and $\mathrm{pid}_{2}$ either. Therefore, there exists an attacker $\overline{\mathcal{A}}$ that violates the forward secrecy or mutual authentication of $\bar{\pi}$. Obviously, $\overline{\mathcal{A}}$ can be easily translated into an attacker $\mathcal{A}$ that successfully attacks $\pi$ which contradicts the assumption.

Therefore, there are at most two connected components, w.l.o.g. pid ${ }_{1}$ and $\mathrm{pid}_{2}$. By the definition of connected components, at least $\mid$ pid $_{1} \mid-1$ messages are sent within pid $_{1}$ and likewise $\mid$ pid $_{2} \mid-1$ messages are sent within pid $_{2}$. This shows that at least $\mid$ pid $_{1}|-1+|$ pid $_{2} \mid-1=n-2$ messages are sent.

Corollary 1. Let $\pi$ denote any GKE with $n \geq 3$ that provides forward secrecy and mutual authentication against insider attackers. Then $\pi$ requires at least two rounds. Furthermore, if $\pi$ is composed of exactly two rounds, then at least $\frac{1}{2} n^{2}+\frac{1}{2} n-3$ messages are required.

Proof. For the first claim, assume that $\pi$ uses only one round. 1-round protocols can be considered as 2-round protocols, where no messages are sent in the first or second round. In the first case, Theorem 2 implies that the number $n$ of parties is at most 2. In the second case, Theorem 1 implies that the number $n$ of parties is at most 2 since there exists at most one pair of parties who does not exchange any messages in the second round. As we consider the case of $n \geq 3$ parties, this yields a contradiction. Hence, $n \geq 3$ implies that at least 2 rounds are required, showing the first claim.

Now consider a 2-round protocol. By Theorem 2, at least $n-2$ messages are necessary in the first round. By Theorem 1, there exists at most one pair who does not exchange any message in the second round. As a consequence, the second round requires at least $\frac{n \cdot(n-1)}{2}-1$ messages. Adding both together yields the second claim.

\section{The Proposed Protocol}

In the following, we propose a 2-round group key exchange protocol which is an extension of the Burmester-Desmedt star-based protocol [12]. It requires $\frac{1}{2} n^{2}+\frac{3}{2} n-2$ messages which is exactly $n+1$ more than the lower bound derived in the previous section. In that sense, our protocol is close to the lower bound and is asymptotically optimal. In Sec. 5 we prove that the protocol is UC-secure according to the model given by Katz and Shin [20].

Before we describe the protocol into details, we give an overview on the basic ideas and its structure. We index each party in pid by $i \in\{1, \ldots, n\}$, that is $\Pi_{i}$ denotes the $i$-th party, and assume that the indices are uniquely determined from pid. Furthermore, we presume that one single party is fixed, w.l.o.g. $\Pi_{1}$. All parties can agree to the same single party without the need of additional communication, for example by choosing the party whose identifier is the first in lexicographical order in pid. Each party $\Pi_{i}$ has its own pair of public/private keys $\left(P K_{i}, S K_{i}\right)$ for signing messages and the public keys are known to all parties. Once a party $\Pi_{j}$ receives a message (sid, pid, new-session), a party instance $\left(\Pi_{j}\right.$, sid, pid) is created. For simplicity we identify the party instances $\left(\Pi_{j}\right.$, sid, pid) with the parties $\Pi_{j}$.

The protocol is conceptually divided into 4 protocols $\pi_{1}, \ldots, \pi_{4}$, that run in parallel. These are briefly explained in Table 1 In protocols $\pi_{1}$ and $\pi_{2}$, all necessary information are distributed that allow for computing the group key $\kappa$. With protocol $\pi_{3}$ every 
party confirms to the others that it accepted to participate into a protocol identified by (sid, pid). This mechanism prevents an adversary from impersonating honest parties. Via protocol $\pi_{4}$, every party signs the commitment com and sends the signature to some other parties according to a distribution schedule. Here, in principle any schedule can be used as long as it guarantees that within each pair of parties, at least one of both receives a signature $\sigma$ from the other. One possible realization is that $\Pi_{i}$ sends the signatures to all $\Pi_{j}$ with $i<j$. This allows the recipient to check if both share the same key. Observe that we do not require that every party gets a signature from every other party. We will show that this is still sufficient for guaranteeing mutual authentication. From our point of view, it is an interesting observation that an asymmetric condition is sufficient for ensuring a symmetric security property.

Table 1. High level description of the four sub-protocols

\begin{tabular}{|c|c|}
\hline \multicolumn{2}{|c|}{ Round 1 } \\
\hline$\pi_{1}$ & $\Pi_{1}$ exchanges a key $x_{j}^{\prime}$ with every other party $\Pi_{j}$. \\
\hline$\pi_{2}$ & $\Pi_{1}$ generates a group key $\kappa$ and sends a commitment com on $\kappa$ to every other party. \\
\hline$\pi_{3}$ & Every party $\Pi_{j}$ for $j \neq i$ generates a signature $\sigma_{j}^{\prime}$ on $($ sid, pid $)$ and sends it to $\Pi_{1}$. \\
\hline
\end{tabular}

\begin{tabular}{|c||c|}
\hline \multicolumn{2}{|c|}{ Round 2 } \\
\hline$\pi_{2}$ & $\Pi_{1}$ sends an encrypted opening of com to each $\Pi_{j \neq 1}$ (using the bilateral keys $x_{j}^{\prime}$ from $\pi_{1}$ ). \\
\hline$\pi_{3}$ & $\Pi_{1}$ distributes the set $\left\{\sigma_{\ell}^{\prime}\right\} \ell$ of signatures to all parties. \\
\hline$\pi_{4}$ & $\begin{array}{c}\text { Every } \Pi_{j} \text { generates a signature } \sigma_{j} \text { on }(\text { sid, pid, com) and sends it to some other parties } \\
\text { according to a predefined distribution schedule. }\end{array}$ \\
\hline
\end{tabular}

\begin{tabular}{|c|c|}
\hline \multicolumn{2}{|c|}{$\begin{array}{c}\text { Key generation } \\
\text { (a party accepts the key } \kappa \text { if } \ldots)\end{array}$} \\
\hline$\pi_{2}$ & com is a commitment on $\kappa$. \\
\hline$\pi_{3}$ & Every $\sigma_{\ell}^{\prime}$ is a valid signature of $\Pi_{\ell}$ on $($ sid, pid). \\
\hline$\pi_{4}$ & $\Pi_{j}$ received all signatures $\sigma_{\ell}$ from $\Pi_{\ell}$ according to the distribution schedule. \\
\hline
\end{tabular}

We now present a concrete description. We use a 1-round 2-party key exchange protocol here, being essentially the Diffie-Hellman key-exchange protocol with authenticated channels where a universal hash function is applied to the result to generate a smooth key. To improve the efficiency of the protocol, $\Pi_{1}$ uses the same ephemeral state $r_{1}$ for all key exchange protocols.

Let (TGen, TCom, TVer, TOpen) be a trapdoor commitment scheme. Given a security parameter $k^{\prime}$, the probabilistic algorithm TGen outputs a pair of parameters and trapdoor (prm, $\tau$ ). Given parameters prm and a message $\mu$, the probabilistic algorithm TCom outputs a pair of commitment and decommitment (com, dec) on the message $\mu$. Given prm, $\mu$, com, and dec, TVer accepts (and outputs (acc)) if com is a correct commitment on $\mu$. Otherwise it rejects and outputs (rej). Given prm, $\mu$, dec, $\tau$, and another message $\mu^{\prime}$, TOpen outputs another decommitment dec ${ }^{\prime}$ such that TVer accepts (prm, $\mu^{\prime}$, com, $\operatorname{dec}^{\prime}$ ). Although the following description of our protocol uses a trapdoor commitment scheme, our protocol also works with commitment schemes that do 
not provide a trapdoor. Actually, the trapdoor is only used to allow straight-line simulatability for the proof of UC-security (Sec. 5).

Let $k, k^{\prime}, m, m^{\prime}, \lambda$ be security parameters, where $k$ is the length of the group key and $m$ is the minimum integer such that $\mathcal{D}$, defined as the space of decommitments dec, is a subset of $\{0,1\}^{m}$. Let $p$ be a prime of size $k^{\prime}+\lambda+1$ and $\mathcal{G}$ be a cyclic group of prime orders $p$ with a generator $g$. Let $\mathcal{U} \mathcal{H}^{\prime}:\{0,1\}^{k^{\prime}} \times\{0,1\}^{m^{\prime}} \rightarrow\{0,1\}^{k+m}$ be a universal hash function [18], $\phi: \mathcal{G} \rightarrow\{0,1\}^{k^{\prime}}$ be a projection, and define $\mathcal{U} \mathcal{H}(z, v):=\mathcal{U H}^{\prime}(\phi(z), v)$. We assume $k^{\prime}$ and $m^{\prime}$ to be sufficiently large so that $(v, \mathcal{U H}(z, v))$ for randomly chosen $z \in \mathcal{G}$ and $v \in\{0,1\}^{m^{\prime}}$ is indistinguishable from $\left(v, x^{\prime}\right)$ when $x^{\prime}$ is randomly distributed in $\{0,1\}^{k+m}$. The necessary size of $k^{\prime}$ and $m^{\prime}$ are determined by the leftover hash lemma [18[19]. The system parameters are $k, k^{\prime}, m, m^{\prime}, \lambda, p, \mathcal{G}, g$, prm, and $\mathcal{U} \mathcal{H}$.

We also require $\mathcal{G}, \phi$ to be chosen such that $\phi^{-1}:\{0,1\}^{k^{\prime}} \rightarrow \mathcal{G}$ can be efficiently computed with overwhelming probability and that the size of $\phi^{-1}(y)$ for randomly chosen $y \in\{0,1\}^{k^{\prime}}$ is sufficiently large. This is possible if $\mathcal{G}$ is an elliptic curve of a prime order $p$ and $\phi$ extracts the least significant $k^{\prime}$ bits of the $x$-coordinate of given point in $\mathcal{G}$ with a large $\lambda$. Then, given a point in $\{0,1\}^{k^{\prime}}$, there are $2^{\lambda}$ possible $x$ coordinates on average. For each candidate, there exists a y-coordinate with probability $1 / 2$ such that this pair is a point on the curve. Thus, if one tries $2^{\lambda}$ possible values in $\mathcal{G}$, an appropriate $z \in \mathcal{G}$ can be found with probability at least $1-1 / 2^{2^{\lambda}}$. Hence, choosing a sufficiently large $\lambda$, an appropriate $\mathcal{G}$ and $\phi$ can be found. This later allows the adversary to find $z \in \mathcal{G}$ such that $(\kappa$, dec $)=\mathcal{U H}(z, v)$ for randomly given $\kappa \in\{0,1\}^{k}$, dec $\in \mathcal{D}$, and $v \in\{0,1\}^{m^{\prime}}$.

At the beginning of the protocol, a value prm is randomly chosen according to the distribution induced by TGen 5 and is given to all parties as a common reference string for the protocol. For the sake of readability, we implicitly assume that all participating parties received (presumably correct) messages according to the protocol and stores all necessary values that are required for a successful protocol run. For example, a party participates only into the protocol if it initially received a (sid, pid, new-session). If this does not happen in a certain time period, the party does not further take part into the protocol without some further actions (e.g., requesting the missing messages, aborting, etc.). The full description of the protocol is given in Fig. 2.

Communication effort. In the first round $\Pi_{1}$ sends $n-1$ messages and each of the other $n-1$ parties send exactly one message, thus $2(n-1)$ messages in total. In the second round, for each pair of parties, exactly one party sends one message to the other, giving $n(n-1) / 2$ messages in the second round. Altogether, $2(n-1)+n(n-1) / 2=$ $\frac{1}{2} n^{2}+\frac{3}{2} n-2$ messages are exchanged in the protocol.

\section{Proof of Security}

In this section we prove that the proposed protocol achieves UC-security according to the model given by Katz and Shin [20]. We suggest this work and [13] for a more detailed description of UC. Informally, a protocol $\pi$ realizes a cryptographic task in an

${ }^{5}$ It is not necessary to execute TGen as long as prm can be sampled from the same distribution. 


\section{Round 1}

$\Pi_{1}$, Computation: $\Pi_{1}$ randomly chooses a group key $\kappa \in\{0,1\}^{k}$ and generates a commitment on these values: $(\mathrm{com}, \mathrm{dec}):=\mathrm{TCom}(\kappa)$. Furthermore, it randomly picks $r_{1} \in \mathbb{Z} / p \mathbb{Z}$ and computes $y_{1}=g^{r_{1}}$. Finally, it samples random values $v_{j} \in\{0,1\}^{m^{\prime}}$, $j=2, \ldots, n$, and generates signatures $\sigma_{1, j}^{\prime}$ on (sid, pid, com, $\left.y_{1}, v_{j}\right)$ for $j=2, \ldots, n$.

$\Pi_{1}$, Storage: $\Pi_{1}$ stores (sid, pid, dec, $\left.\kappa,\left(v_{j}\right)_{j=2, \ldots, n}, r_{1}\right)$.

$\Pi_{1}$, Communication: $\Pi_{1}$ sends to each $\Pi_{j} \in$ pid $\backslash\left\{\Pi_{1}\right\}$ the message $\left(\Pi_{1} ;\right.$ sid, pid, com, $\left.y_{1}, v_{j}, \sigma_{1, j}^{\prime}\right)$

$\Pi_{\mathbf{i}} \neq \Pi_{\mathbf{1}}$, Computation: $\Pi_{i}$ randomly chooses $r_{i} \in \mathbb{Z} / p \mathbb{Z}$ and computes $y_{i}=g^{r_{i}}$. Then $\Pi_{i}$ generates a signature $\sigma_{i}^{\prime}$ on (sid, pid, $\left.y_{i}\right)$.

$\Pi_{\mathbf{i}} \neq \Pi_{\mathbf{1}}$, Storage: $\Pi_{i}$ stores (sid, pid, $\left.r_{i}\right)$.

$\Pi_{\mathbf{i}} \neq \Pi_{1}$, Communication: $\Pi_{i}$ sends $\left(\Pi_{i} ;\right.$ sid, pid, $\left.y_{i}, \sigma_{i}^{\prime}\right)$ to $\Pi_{1}$.

\section{Round 2}

$\Pi_{1}$, Computation: $\Pi_{1}$ verifies that each $\sigma_{j}^{\prime}$ is a valid signature on (sid, pid, $\left.y_{j}\right)$ by $\Pi_{j}$ and generates $z_{j}=y_{j}{ }^{r_{1}}, x_{j}=(\kappa, \mathrm{dec}) \oplus \mathcal{U} \mathcal{H}\left(z_{j}, v_{j}\right) . \Pi_{1}$ also generates a signature $\sigma_{1}$ on (sid, pid, com).

$\Pi_{1}$, Storage: $\Pi_{1}$ updates its storage to (sid, pid, com, $\kappa$ ).

$\Pi_{1}$, Communication: $\Pi_{1}$ sends $\left(\Pi_{1} ;\right.$ sid, pid,com, $\left.\sigma_{1}\right), x_{j}$, and $\left(\sigma_{\ell}^{\prime}, y_{\ell}\right)_{\ell>1}$ to each $\Pi_{j} \neq \Pi_{1}$.

$\Pi_{\mathbf{i}} \neq \Pi_{\mathbf{1}}$, Computation: $\Pi_{i}$ verifies that $\sigma_{1, i}^{\prime}$ is a valid signature on (sid, pid, com, $y_{1}, v_{i}$ ) by $\Pi_{1}$ and generates $z_{i}=y_{1}{ }^{r_{i}}, x_{i}^{\prime}=\mathcal{U} \mathcal{H}\left(z_{i}, v_{i}\right)$ and a signature $\sigma_{i}$ on (sid, pid,com).

$\Pi_{\mathbf{i}} \neq \Pi_{\mathbf{1}}$, Storage: $\Pi_{i}$ updates its storage to (sid, pid, com, $x_{i}^{\prime}$ ).

$\Pi_{\mathbf{i}} \neq \Pi_{1}$, Communication: $\Pi_{i}$ sends $\left(\Pi_{i} ;\right.$ sid, pid, com, $\left.\sigma_{i}\right)$ to all $\Pi_{j} \in$ pid specified by the distribution schedule.

\section{Key generation}

$\Pi_{1}$, Computation: If every $\sigma_{j}$ is a valid signature on (sid, pid, com) by $\Pi_{j}$, then $\Pi_{1}$ outputs (sid, pid, $\kappa)$ and deletes its storage (instance state).

$\Pi_{\mathbf{i}} \neq \boldsymbol{\Pi}_{\mathbf{1}}$, Computation: If all received signatures are correct, then it computes $\left(\kappa_{i}, \operatorname{dec}_{i}\right)=$ $x_{i}^{\prime} \oplus x_{i}$. If acc $=\operatorname{TVer}\left(\mathrm{prm}, \kappa_{i}, \operatorname{com}, \operatorname{dec}_{i}\right)$, then $\Pi_{i}$ outputs $($ sid, pid, $\kappa)$ and deletes its state.

Fig. 2. The protocol

UC-secure way if no pair of environment $\mathcal{Z}$ and real adversary $\mathcal{A}$, can distinguish the protocol execution in the real world, called real execution, from the execution of an ideal functionality $\mathcal{F}$ in an ideal world, called ideal execution. $\mathcal{F}$ can be seen as a kind of black box that ideally realizes the considered cryptographic task. We recall in Fig. 3 the definition of its ideal functionality for GKEs from Katz and Shin [20]. 


\section{Ideal Functionality $F_{G K E}$}

([20]) The ideal functionality $\mathcal{F}_{\mathrm{GKE}}$ interacts with parties $\Phi_{1}, \ldots, \Phi_{n}$ and an ideal adversary $\mathcal{S} . \mathcal{F}_{\mathrm{GKE}}$ runs on a security parameter $k$.

Initialization: Upon receiving (sid, pid, new-session) from a party $\Phi_{i}$ for the first time (where $\mid$ pid $\mid \geq 2$ ), $\mathcal{F}_{\mathrm{GKE}}$ records (sid, pid, $\Phi_{i}$ ) and sends this to $\mathcal{S}$. Once the tuples (sid, pid, $\Phi_{j}$ ) for all $\Phi_{j} \in$ pid are recorded, $\mathcal{F}_{\mathrm{GKE}}$ stores (sid, pid, ready) and sends it to $\mathcal{S}$.

Key generation: Upon receiving a message (sid, pid, ok) from $\mathcal{S}$ where there is a recorded tuple (sid, pid, ready), it checks if all $\Phi_{j} \in$ pid are uncorrupted. If this is the case, it chooses uniformly a key $\kappa \in\{0,1\}^{k}$ and stores (sid,pid, $\kappa$ ). If any of the parties $\Phi_{j} \in$ pid is corrupted, it waits for $\mathcal{S}$ to send a message (sid, pid, key, $\kappa$ ) and then stores (sid, pid, $\kappa$ ).

Key delivery: If $\mathcal{S}$ sends a message (sid, pid, deliver, $\left.\Phi_{i}\right)$ where there is a recorded tuple (sid, pid, $\kappa$ ) and $\Phi_{i} \in$ pid, then $\mathcal{F}_{\mathrm{GKE}}$ sends (sid, pid, $\kappa$ ) to party $\Phi_{i}$.

Player corruption: If $\mathcal{S}$ corrupts $\Phi_{i} \in$ pid where there is a recorded tuple (sid, pid, $\kappa$ ) but a message (sid, pid, $\kappa$ ) has not yet been sent to $\Phi_{i}$, then $\mathcal{S}$ is given $\kappa$, otherwise nothing.

Fig. 3. The group key exchange functionality

Theorem 3. The protocol described in Sec. 4 UC-realizes the ideal functionality of GKE if the used signature scheme is secure and if the decisional Diffie-Hellman assumption holds in the common reference string model.

For the proof, we have to give an ideal adversary $\mathcal{S}$ such that no pair of environment $\mathcal{Z}$ and real adversary $\mathcal{A}$ can tell apart a real execution (i.e., real parties $\Pi_{i}$ executing the protocol) and an ideal execution (i.e., $\mathcal{S}$ simulates parties $\Pi_{i}^{\mathcal{S}}$ compatibly to the outputs of the ideal functionality $\mathcal{F}:=\mathcal{F}_{\text {GKE }}$ that in turn communicates with ideal parties $\Phi_{i}$ ).

We first define an ideal adversary $\mathcal{S}$ and show afterward in Lemmas 1 and 2 that it fulfills the condition from above. $\mathcal{S}$ has black box access to the real adversary $\mathcal{A}$. Messages from $\mathcal{Z}$ to $\mathcal{S}$ ( $\mathcal{Z}$ believes it is sending to $\mathcal{A}$ ) are forwarded to $\mathcal{A}$ and vice versa. Additionally, $\mathcal{S}$ simulates the real parties $\Pi_{i}$ on behalf of all uncorrupted ideal parties $\Phi_{i}$. The simulated parties are denoted by $\Pi_{i}^{\mathcal{S}}$. As opposed to the ideal parties, the simulated parties participate in a protocol execution. At the beginning, $\mathcal{S}$ generates for every $\Pi_{j}^{\mathcal{S}}$ a public/private key pairs $\left(P K_{j}, S K_{j}\right)$ for digital signatures and gives the public keys to $\mathcal{A}$. Any messages sent by $\mathcal{A}$ to $\Pi_{i}$ are processed by $\Pi_{i}^{\mathcal{S}}$, and any messages output by $\Pi_{i}^{\mathcal{S}}$ are given to $\mathcal{A}$. $\mathcal{S}$ also runs TGen $\left(k^{\prime}\right)$ for a security parameter $k^{\prime}$ to obtain $($ prm, $\tau$ ). prm is the common reference string of the protocol.

In addition to the above, the ideal adversary $\mathcal{S}$ proceeds as follows:

Session Key Generation (SKG): If at any point in time a simulated party $\Pi_{i}^{\mathcal{S}}$ outputs a key $(\operatorname{sid}, p i d, \kappa), \mathcal{S}$ checks to see whether any of the parties in pid have been corrupted.

SKG. $\neg$ cor.: If no parties in pid are corrupted, then:

SKG. $\neg$ cor. $\neg$ ok: If $\mathcal{S}$ has not yet sent (sid, pid, ok) to $\mathcal{F}$, then $\mathcal{S}$ checks that it has received the message (sid, pid, ready) from $\mathcal{F}$. If not, $\mathcal{S}$ aborts. Otherwise, it sends to $\mathcal{F}$ the messages (sid, pid, ok) and (sid, pid, deliver, $\Phi_{i}$ ). 
SKG. $\neg$ cor.ok: If $\mathcal{S}$ has already sent the message (sid, pid, ok) to $\mathcal{F}$, then $\mathcal{S}$ sends the message (sid, pid, deliver, $\Phi_{i}$ ) to $\mathcal{F}$.

SKG.cor.: Otherwise, say $C \subseteq$ pid $\backslash\left\{\Phi_{i}\right\}$ are corrupted. Then:

SKG.cor. $\neg$ ok: If $\mathcal{S}$ has not yet sent (sid, pid, ok) to $\mathcal{F}$, then it sends on behalf of all $\Phi_{j} \in C$ the message (sid, pid, new-session, $\Phi_{j}$ ) to $\mathcal{F}$ who have not done so already, receives (sid, pid, ready) from $\mathcal{F}$, and then sends (sid, pid, ok) to $\mathcal{F}$. (If $\mathcal{S}$ does not receive (sid, pid, ready) after executing the above, it aborts.) Next, $\mathcal{S}$ sends (sid, pid, key, $\kappa$ ) and (sid, pid, deliver, $\left.\Phi_{i}\right)$ to $\mathcal{F}$.

SKG.cor.ok: If $\mathcal{S}$ has already sent (sid, pid, ok) to $\mathcal{F}$, then $\mathcal{S}$ checks that no parties in pid were corrupted at that point in time.

1. If this is the case, then $\mathcal{S}$ sends (sid, pid, deliver, $\Phi_{i}$ ) to $\mathcal{F}$.

2. Otherwise, $\mathcal{S}$ has already sent (sid, pid, key, $\left.\kappa^{\prime}\right)$ to $\mathcal{F}$ (i.e., a party in pid was corrupted at the time the "ok" message was sent). If $\kappa^{\prime} \neq \kappa$ then $\mathcal{S}$ aborts. Otherwise, $\mathcal{S}$ sends the message (sid, pid, deliver, $\Phi_{i}$ ) to $\mathcal{F}$.

Corruption (COR): When $\mathcal{A}$ intends to corrupt a party $\Phi_{i}, \mathcal{S}$ corrupts that party in the ideal world. $\mathcal{S}$ also provides $\mathcal{A}$ with the secret key $S K_{i}$ and the current internal state of $\Phi_{i}$ as follows:

COR. $\neg$ ok: If $\mathcal{S}$ has not yet sent (sid,pid, ok) to $\mathcal{F}$, then $\mathcal{S}$ simply gives $\mathcal{A}$ the current instance state of $\Pi_{i}^{\mathcal{S}}$ if it exists.

COR.ok: Otherwise $\mathcal{S}$ has already sent (sid, pid, ok) to $\mathcal{F}$. Then:

COR.ok. $\neg$ del: If $\mathcal{S}$ has not yet sent (sid, pid, deliver, $\Phi_{i}$ ) to $\mathcal{F}$, then it checks if $\Pi_{i}^{\mathcal{S}}$ did finish the first round of the protocol. If this is not the case, then $\mathcal{S}$ aborts. Otherwise, $\mathcal{S}$ corrupts $\Phi_{i}$ to obtain a key $\kappa$ from $\mathcal{F}$. This might either been provided by $\mathcal{S}$ before or been generated by $\mathcal{F}$. In the first case, $\mathcal{S}$ simply forwards the internal state of $\Pi_{i}^{\mathcal{S}}$ to $\mathcal{A}$. In the second case, we make use of the fact that $\mathcal{S}$ has already sent (sid,pid, ok). This implies either of the following:

$\neg \boldsymbol{\Phi}_{\mathbf{1}}$ : When $i \neq 1$, at least $\Phi_{1}$ in pid has already sent $x_{i}$ to $\Phi_{i}$. $\mathcal{S}$ uses the trapdoor $\tau$ to generate a fitting decommitment dec, that is dec $=$ TOpen $\left(\operatorname{prm}, \kappa^{\prime}, \operatorname{dec}^{\prime}, \tau, \kappa\right)$. Here, $\left(\kappa^{\prime}, \operatorname{dec}^{\prime}\right)$ are the values generated by $\Pi_{1}^{S}$, i.e., $\left(\mathrm{com}, \mathrm{dec}^{\prime}\right)=\mathrm{TCom}\left(\mathrm{prm}, \kappa^{\prime}\right)$. Then, $\mathcal{S}$ generates $x_{i}^{\prime}=$ $x_{i} \oplus(\kappa, \mathrm{dec})$ and hands to $\mathcal{A}$ the instance state (sid, pid, com, $\left.x_{i}^{\prime}\right)$. We note it is possible to find $z_{i}$ such that $x_{i}^{\prime}=\mathcal{U} \mathcal{H}\left(z_{i}, v_{i}\right)$ with overwhelming probability if $\lambda$ is long enough.

$\boldsymbol{\Phi}_{\mathbf{1}}$ : When $i=1, \mathcal{S}$ hands to $\mathcal{A}$ the instance state (sid, pid, com, $\kappa$ ).

COR.ok.del: If $\mathcal{S}$ has already sent (sid, pid, deliver, $\Phi_{i}$ ) to $\mathcal{F}$, then $\mathcal{S}$ returns nothing (i.e., an empty instance state) to $\mathcal{A}$.

Next we show that the ideal adversary $\mathcal{S}$ aborts only with negligible probability (Lemma 1 ) and that the real and the ideal execution are indistinguishable (under the decisional Diffie-Hellman assumption) if $\mathcal{S}$ does not abort (Lemma 2). From these two Lemmas, Theorem 3 follows immediately.

Lemma 1. The probability that the ideal adversary $\mathcal{S}$ defined above aborts is negligible. 
Proof. Suppose that there exists a real adversary $\mathcal{A}$ that can make the ideal adversary $\mathcal{S}$ abort. By the description of $\mathcal{S}$, there are exactly four situation when this might happen: SKG. $\neg$ cor. $\neg$ ok, SKG.cor. $\neg$ ok. COR.ok. $\neg$ del, and SKG.cor.ok.

Assume that the abort has happened in one of the two cases SKG. $\neg$ cor. $\neg$ ok or SKG.cor. $\neg$ ok. In both cases, a simulated party $\Pi_{i}^{S}$ did output (sid,pid, $\kappa$ ) (session key generation) but $\mathcal{S}$ did not receive (sid, pid, ready) from $\mathcal{F}$. This in turn means that there exists at least one uncorrupted simulated party $\Pi_{j}^{S} \in$ pid such that $\mathcal{Z}$ never sent (sid, pid, new-session). Hence, $\Pi_{j}^{S}$ has never signed any message. On the other hand, by the protocol specification $\Pi_{i}^{\mathcal{S}}$ only generates the key if it received a valid signature from all parties. Therefore, this case can only happen if $\mathcal{A}$ forged a signature.

Suppose now that the abort took place in the case of COR.ok. $\neg$ del. This means that on the one hand $\mathcal{S}$ has send before the message (sid, pid, ok) and hence there exists an uncorrupted simulated party $\Pi_{j}^{S} \in$ pid who has output (sid, pid, $\kappa$ ). On the other hand, the abort condition tells that there exists a simulated party $\Pi_{i}^{\mathcal{S}}$ that has not finished the first round at this point in time. In particular, $\Pi_{i}^{\mathcal{S}}$ had not send a signed message so far. With the same arguments as above, this can only happen if $\mathcal{A}$ forged a signature.

It remains to consider the final case: SKG.cor.ok. By definition $\mathcal{S}$ has to deal only with key generation if the key is generated by an uncorrupted party. The abort condition says in principle that two uncorrupted parties $\Pi_{i}$ and $\Pi_{j}$ have generated two different keys $\kappa$ and $\kappa^{\prime}$, respectively. Recall that the key is reconstructed from a commitment distributed by $\Pi_{1}$. As the commitment has been signed by $\Pi_{1}$ and as at least one of the two parties can check if both received the same commitment, it must hold that $\Pi_{i}$ and $\Pi_{j}$ received the same commitment com (as none of them aborted) unless $\mathcal{A}$ has forged a signature. But then the binding property of the commitment schemes implies that $\kappa=\kappa^{\prime}$ what contradicts the assumption. Hence, this case can also only happen in the case of a signature forgery.

Lemma 2. Suppose that the ideal adversary does not abort. Then, for any environment and any real adversary, the ideal execution and the real execution are indistinguishable as long as the decisional Diffie-Hellman assumption holds in the standard model.

Proof. Suppose that there exists an environment $\mathcal{Z}$ that can distinguish between the ideal execution and the real execution. We will first show that $\mathcal{Z}$ does not gain any useful information by corrupting parties. As a consequence we can restrict w.l.o.g. to an environment $\mathcal{Z}^{\prime}$ that does no corruption at all. Finally, we will construct an algorithm $\mathcal{B}$ from $\mathcal{Z}^{\prime}$ that allows for solving the decisional Diffie-Hellman problem.

Assume that $\mathcal{Z}$ corrupts a party before any key has been generated. By definition of $\mathcal{S}$, from this point on $\mathcal{Z}$ only communicates with the simulated parties (e.g., learns its states after corruption) and receives the results of the simulated protocol run. In other words, the ideal functionality $\mathcal{F}$ is no longer involved. Obviously, in this case the real and ideal executions are indistinguishable as the simulated parties behave exactly like real parties.

Now we turn our attention to the case that $\mathcal{Z}$ only corrupts after a key has been generated. By definition, this means that the key $\kappa^{\prime}$ of the simulated protocol run has

\footnotetext{
${ }^{6}$ Observe that the trapdoor $\tau$ is known only to $\mathcal{S}$ but not to any of the parties.
} 
been replaced by a key $\kappa$ that has been given by the ideal functionality. In particular all players have finished both rounds already (the information send around at the end of round 2 are a necessary pre-requisite for key generation). In the case that $\Pi_{1}$ gets corrupted, $\mathcal{Z}$ receives the values (sid, pid, com, $\kappa$ ) (where $\kappa$ is the key generated by $\mathcal{F}$ ). This is obviously a perfect simulation. In the case that $\Pi_{j} \neq \Pi_{1}$ gets corrupted, the situation becomes a little bit more tricky: besides the values mentioned above, a real party would additionally store an encryption of a decommitment $\mathrm{dec}^{\prime}$ that opens the distributed commitment com to the "real" key $\kappa^{\prime}$. Here we make use of the trapdoor in the commitment scheme and replace dec' $^{\prime}$ by another decommitment dec that opens com to $\kappa$ (instead of $\kappa^{\prime}$ ). By the definition of the commitment scheme, this yields a perfect simulation as well.

Concluding, an environment $\mathcal{Z}$ does not observe any differences between both executions via corruption. Thus, we can restrict to an environment $\mathcal{Z}^{\prime}$ that does not corrupt at all. We construct from $\mathcal{Z}^{\prime}$ an algorithm $\mathcal{B}$ that breaks the decisional Diffie-Hellman assumption. That is given a triple $(g, a, b, c)=\left(g, g^{\alpha}, g^{\beta}, g^{\gamma}\right)$, we construct an algorithm $\mathcal{B}$ (based on $\mathcal{Z}^{\prime}$ ) that decides whether $\alpha \cdot \beta=\gamma$ or not.

$\mathcal{B}$ behaves in principle like the ideal adversary $\mathcal{S}$ except of the following difference. $\mathcal{B}$ replaces the values occurring within the Diffie-Hellman key exchange by other parameters that are derived from the problem instance mentioned above as follows:

$$
y_{1} \leftarrow b, \quad y_{j} \leftarrow g^{\mu_{j}} a^{\nu_{j}}=g^{\mu_{j}+\alpha \cdot \nu_{j}}, \quad z_{j} \leftarrow b^{\mu_{j}} c^{\nu_{j}}=g^{\beta \cdot \mu_{j}+\gamma \cdot \nu_{j}} .
$$

where $\left\{\mu_{j}, \nu_{j} \in \mathbb{Z} / p \mathbb{Z}\right\}_{j=1, \ldots, n}$ are randomly chosen. The fact that $\mathcal{B}$ does not know the discrete logarithms of these values (as opposed to the real execution) is not a problem as $\mathcal{Z}^{\prime}$ does not corrupt by assumption. Observe that $z_{j}$ is a Diffie-Hellman key derived from $y_{1}$ and $y_{j}$ if and only if $\alpha \cdot \beta=\gamma$. Therefore, if $\alpha \cdot \beta=\gamma$, then $\mathcal{B}$ acts exactly like $\mathcal{S}$. By assumption $\mathcal{Z}^{\prime}$ can distinguish between the ideal or real execution in this case. However, if $\alpha \cdot \beta \neq \gamma$ then the messages of the parties are independent of the key $\kappa$. Thus, $\mathcal{Z}^{\prime}$ cannot have any advantage in this case. Now, $\mathcal{B}$ invokes $\mathcal{Z}^{\prime}$ several times with different values of $\mu_{j}$ and $\nu_{j}$ and estimates the advantage of $\mathcal{Z}^{\prime}$. If this is negligible, then $\mathcal{B}$ assumes that $\alpha \cdot \beta \neq \gamma$. Otherwise it guesses that $\alpha \cdot \beta=\gamma$. With the arguments above, this yields a distinguisher for the decisional Diffie-Hellman problem.

Concluding we have seen that (under the decisional Diffie-Hellman assumption) that for none of the three cases an environment can exist that efficiently distinguishes between both executions.

\section{Conclusion}

We addressed the question of the communication complexity in group key exchange (GKE) protocols. We derived from basic security requirements, i.e., forward secrecy and mutual authentication, that any GKE needs at least two rounds and a lower bound on the number of messages for this case. Furthermore we presented a UC-secure protocol that almost achieves these bounds.

Still, several open questions remain. Some of them are: (1) Is it possible to either construct protocols that exactly meet these bounds or can it be proved that the minimum effort is actually higher? (2) Can we determine the effort for additional requirements, 
e.g., contributiveness, etc.? (3) Are UC-secure protocols possible with the same communication effort as our protocol but within the standard model? (4) Are less messages possible if one considers GKEs with more than 2 rounds?

\section{References}

1. Abdalla, M., Catalano, D., Chevalier, C., Pointcheval, D.: Password-authenticated group key agreement with adaptive security and contributiveness. In: Preneel, B. (ed.) AFRICACRYPT 2009. LNCS, vol. 5580, pp. 254-271. Springer, Heidelberg (2009)

2. Bellare, M., Pointcheval, D., Rogaway, P.: Authenticated key exchange secure against dictionary attacks. In: Preneel, B. (ed.) EUROCRYPT 2000. LNCS, vol. 1807, pp. 139-155. Springer, Heidelberg (2000)

3. Bellare, M., Rogaway, P.: Entity authentication and key distribution. In: Stinson, D.R. (ed.) CRYPTO 1993. LNCS, vol. 773, pp. 232-249. Springer, Heidelberg (1994)

4. Bellare, M., Rogaway, P.: Provably secure session key distribution: the three party case. In: STOC, pp. 57-66. ACM, New York (1995)

5. Bohli, J.-M., Vasco, M.I.G., Steinwandt, R.: Secure group key establishment revisited. Int. J. Inf. Sec. 6(4), 243-254 (2007)

6. Bondy, J.A., Murty, U.S.R.: Graph theory with applications. North-Holland, Amsterdam (1976)

7. Bresson, E., Chevassut, O., Pointcheval, D.: Provably authenticated group diffie-hellman key exchange - the dynamic case. In: Boyd, C. (ed.) ASIACRYPT 2001. LNCS, vol. 2248, pp. 290-309. Springer, Heidelberg (2001)

8. Bresson, E., Chevassut, O., Pointcheval, D.: Dynamic group diffie-hellman key exchange under standard assumptions. In: Knudsen, L.R. (ed.) EUROCRYPT 2002. LNCS, vol. 2332, pp. 321-336. Springer, Heidelberg (2002)

9. Bresson, E., Chevassut, O., Pointcheval, D., Quisquater, J.-J.: Provably authenticated group diffie-hellman key exchange. In: ACM Conference on Computer and Communications Security, pp. 255-264 (2001)

10. Bresson, E., Manulis, M.: Securing group key exchange against strong corruptions. In: ASIACCS, pp. 249-260 (2008)

11. Bresson, E., Manulis, M., Schwenk, J.: On security models and compilers for group key exchange protocols. In: Miyaji, A., Kikuchi, H., Rannenberg, K. (eds.) IWSEC 2007. LNCS, vol. 4752, pp. 292-307. Springer, Heidelberg (2007)

12. Burmester, M., Desmedt, Y.: A secure and efficient conference key distribution system. In: De Santis, A. (ed.) EUROCRYPT 1994. LNCS, vol. 950, pp. 275-286. Springer, Heidelberg (1995)

13. Canetti, R.: Universally composable security: A new paradigm for cryptographic protocols. Cryptology ePrint Archive, Report 2000/067 (2000), http: / / eprint.iacr.org/(revised in 2005)

14. Canetti, R.: Universally composable security: A new paradigm for cryptographic protocols. In: FOCS, pp. 136-145 (2001)

15. Furukawa, J., Armknecht, F., Kurosawa, K.: A universally composable group key exchange protocol with minimum communication effort. In: Ostrovsky, R., De Prisco, R., Visconti, I. (eds.) SCN 2008. LNCS, vol. 5229, pp. 392-408. Springer, Heidelberg (2008)

16. Gorantla, M.C., Boyd, C., González Nieto, J.M.: Modeling key compromise impersonation attacks on group key exchange protocols. In: Jarecki, S., Tsudik, G. (eds.) PKC 2009. LNCS, vol. 5443, pp. 105-123. Springer, Heidelberg (2009) 
17. Gorantla, M.C., Boyd, C., Nieto, J.M.G.: Universally composable contributory group key exchange. In: ASIACCS, pp. 146-156 (2009)

18. Impagliazzo, R., Levin, L.A., Luby, M.: Pseudo-random generation from one-way functions (extended abstracts). In: STOC, pp. 12-24. ACM, New York (1989)

19. Impagliazzo, R., Zuckerman, D.: How to recycle random bits. In: FOCS, pp. 248-253. IEEE, Los Alamitos (1989)

20. Katz, J., Shin, J.S.: Modeling insider attacks on group key-exchange protocols. In: CCS 2005: Proceedings of the 12th ACM Conference on Computer and Communications Security, pp. 180-189. ACM Press, New York (2005)

\section{A Forward Secrecy and MA Security}

In this section, we formally define the security notions that are considered in this paper. The attacker's capabilities are modeled by a set of queries that she is allowed to do. These are defined in the following definition.

Definition 1 (Attacker Model). An attacker $\mathcal{A}$ can make the following queries:

Invoke $(\Pi$, sid, pid, new-session): A new instance ( $\Pi$, sid, pid) of $\Pi$ is invoked by sending a message (sid, pid, new-session) to $\Pi$. $\mathcal{A}$ obtains the response of $\Pi$. We assume here that, if a party $\Pi \in$ pid is uncorrupted, that is $\mathcal{A}$ has never queried Corrupt $(\Pi)$ (see below), $\Pi$ is never given the same triple (sid, pid, new-session) more than once.

$\operatorname{Send}(\Pi ; M)$ : The message $M$ is sent to $\Pi$ and $\mathcal{A}$ receives the response.

$\operatorname{RevealKey}(\Pi$, sid, pid): If $\Pi$ has already generated an output $(\Pi$, sid, pid, $\kappa), \mathcal{A}$ is given $\kappa$.

$\operatorname{Corrupt}(\Pi)$ : The long-term secret of $\Pi$ is given to $\mathcal{A}$.

Observe that the queries Invoke and Send model the attacker's capability of controlling the schedules of the parties and the communication in the network. We do not need to consider the strong corruption model where the adversaries are able to obtain instance states. The above weak adversaries are sufficient for our lower bounds.

For defining AKE-security, we introduce an additional query:

Definition 2. The query $\operatorname{Test}(\Pi$, sid, pid) is defined as follows. When $\Pi$ has output $(\Pi$, sid, pid, $\kappa), b \in\{0,1\}$ is randomly chosen. Then, $\mathcal{A}$ is either given $\kappa$ if $b=1$, or $a$ randomly chosen key if $b=0$.

We say that the query $\mathbf{T e s t}(\Pi$, sid, pid) is fresh if the following conditions hold for every $\Pi^{\prime} \in$ pid:

- No query RevealKey $\left(\Pi^{\prime}\right.$, sid, pid $)$ is made after $\Pi^{\prime}$ outputs $\left(\Pi^{\prime}\right.$, sid, pid, $\left.\kappa^{\prime}\right)$ for some $\kappa^{\prime}$.

- No query Corrupt $\left(\Pi^{\prime}\right)$ is made before $\Pi^{\prime}$ outputs $\left(\Pi^{\prime}\right.$, sid, pid, $\left.\kappa^{\prime}\right)$ for some $\kappa^{\prime}$.

Definition 3. We say that an external-SID GKE is AKE-secure if, for every poly-time adversary $\mathcal{A}$, the difference between the probability that $\mathcal{A}$ wins the following game and $1 / 2$ is negligible in some security parameter: 


AKE game:
After $\mathcal{A}$ and the parties are initialized by input of a security parameter, $\mathcal{A}$ interacts
with the parties by making queries as specified in Definition $\Pi \mathcal{A}$ is allowed to
make one query Test $(\Pi$, sid, pid $)$ for some $\Pi$, sid, and pid such that $\Pi$ has output
$(\Pi$, sid, pid, $\kappa)$ once during the game under the condition that Test $(\Pi$, sid, pid)
remains to be fresh until the end of the game. $\mathcal{A}$ outputs a bit $b^{\prime} \in\{0,1\}$ when
the game ends. We say $\mathcal{A}$ wins the game if $b=b^{\prime}$, where $b$ is the bit that oracle
generated when $\mathcal{A}$ queried $\mathbf{T e s t}(\Pi$, sid, pid $)$.

Observe that the freshness requirements guarantees that $\mathcal{A}$ cannot simply obtained knowledge about the key by querying RevealKey or Corrupt. Although adversaries are allowed to corrupt parties after they output the target keys, the above AKE security requires that this faculty does not help adversary to guess the target key. This is the Forward secrecy.

Definition 4. We say an external-SID GKE provides mutual authentication in presence of insider attackers if, for every poly-time adversary $\mathcal{A}$, the probability that $\mathcal{A}$ wins the following game is negligible:

\section{MA game:}

After $\mathcal{A}$ and the parties are initialized by input of a security parameter, $\mathcal{A}$ interacts with the parties by making queries as defined in Definition 1 We say that $\mathcal{A}$ wins the game if two uncorrupted parties $\Pi$ and $\Pi^{\prime}$ output $(\Pi$, sid,pid, $\kappa)$ and $\left(\Pi^{\prime}\right.$, sid, pid, $\left.\kappa^{\prime}\right)$, respectively, such that $\kappa \neq \kappa^{\prime}$ before $\mathcal{A}$ stops. 